\title{
Study on Framework Design of Shore Defense Radar Condition-based Support System based on OSA-CBM
}

\author{
Bing Chen ${ }^{1, a}$, Gang Lu ${ }^{2, b}$, Qian Qian Liu ${ }^{1, c}$ \\ ${ }^{1}$ Naval university of engineering, Wuhhan, 430033, China \\ ${ }^{2}$ Naval Armaments Department, Beijig, 100055, China \\ aemail: chenbing0710@126.com, bemail:Ig19700709@126.com, 'email:liuhe1984@163.com
}

Keywords: Shore Defense Rradar; Condition-based Maintenance; OSA-CBM; Fault Diagnosis and Prediction

\begin{abstract}
With the daily growing position of coast radar in the shore defense system, maintenance is more and more onerous. The traditional assurance mode is hard to meet the accurate and timely need. Learn from the advanced experience of foreign developed countries in the field of equipment maintenance support, the article researches the function framework of shore defense radar condition-based support system reference to the open systems architecture of OSA-CBM, decomposes the technical difficulties and working prospect deeply to make beneficial exploration for improving the shore defense radar support ability of our country.
\end{abstract}

\section{Introduction}

As an important part of shore defense system for protecting homeland security, shore defense radar plays an important role in the task of moving targets detection and gun spotting in the sea level and low-altitude. Poor working conditions and long-term operation fatigue are the main reasons causing shore defense radar fault. In order to guarantee the use effectiveness of combat, most of the equipments adopt the combination of regular maintenance and later maintenance presently ${ }^{[1]}$. Such maintenance model needs large amount of work and human input, predictive maintenance is not enough and exits "repair" and "neglect" phenomenon.

With the growing complexity of weapons and equipment in the modern high technology war, and the daily expanding influence of weapon equipment performance troops fighting capacity keeping, improving equipment reliability, maintainability, supportability and reducing the life cycle cost has become a very urgent request ${ }^{[2]}$. This article refers to the current international advanced security concept and the open systems architecture of OSA-CBM, combines the specific characteristics of shore defense radar, improves adaptability and researches the component architecture to make beneficial exploration on intelligent support and visual maintenance direction.

\section{Demand analysis of condition-based support}

Study the component architecture of condition-based support system of shore defense radar; it needs the composition characteristics and working condition as the basis, data acquisition ability and common failure mode as the focus.

\section{BITE Ability Analysis.}

Recently, BIT achieves remarkable improvement from theory to application, which has been the effective way to improve testability and maintenance of complex system ${ }^{[3]}$. A certain type of radar as a fully digital radar, the designed BITE can scan 67 key parameters, including the key state information, working voltage, the key signal, and etc.; through these parameter information, it can evaluate the work state of radar, support local storage of data and offline analysis.

Data processor assigns scan addresses for each of the scanning parameters independently, when receiving the external communication instructions, such as portable fault detector, the local scanning and query software, etc. Data processor chooses signal channel according to the scan address, achieves signal detection and output according to the communication protocol to get 
internal test information ${ }^{[4]}$.

The data output of BITE has two methods:

1) Scann the query and the local store. During the work of the radar, output the scanning query instruction according to the scanning query software running in the display controlling unit; make cycling scanning query for 67 key parameters, query result is stored in the data processor, using external storage medium, such as USB, exports all data;

2) Maintenance query display. After data processor receives communication command sent by the display controlling unit, set up communication channels with portable fault detector, and sends data scanning address parameter pointed by communication instructions to portable fault detector for display and analysis.

\section{Common fault modes.}

According to the fault compiled statistics of a certain type of shore radar, common fault modes of equipment level of shore radar include:

1) Network fault;

2) No target output;

3) Output outside the target without Angle;

4) No simulation target.

Specifically, vulnerable parts and fault types mainly include:

1) Voltage fault, such as $48 \mathrm{~V}$ voltage, $24 \mathrm{~V}$ voltage, $96 \mathrm{~V}$ voltage fault;

2) Signal fault, such as azimuth jump code, modulating pulse I, II, III, output fault, clock fault, and high I fault;

3) Communication fault, such as servo unit communication is not normal, network failure, and so on;

4) Electro-mechanical fault, such as cooling fan of travelling wave tube stalls; speed is inconsistent under manual mode; servo unit is rotating after combining with electric, and so on.

\section{System framework of condition-based maintenance}

\section{System Framework of OSA-CBM.}

Face the increasingly complexity, high automation and information of equipment, traditional maintenance mode increasingly exposed shortcomings. With the development of testing technology, condition monitoring technology, fault diagnosis technology and maintenance analysis decision technology, appears the maintenance (CBM) looking condition based monitoring and fault diagnosis technology as the basis based on state. It predicts the lifetime correctly to determine maintenance needs according to the monitoring of the equipment state.

The definition of US Army is that CBM is through the built-in sensors or portable external equipment testing, is a maintenance process of real-time evaluation for system state ${ }^{[5]}$.

According to the function, the system architecture of CBM can be classified as: data acquisition, signal processing, condition monitoring, condition assessment, prediction, decision support and presentation layer $7^{[6]}$, is shown in figure 1 .

The establishment and promotion of CBM system can prevent the accident, avoid catastrophic losses caused by unexpected fault; Can significantly improve the economic benefit, reduce unnecessary downtime, optimize the operating life of equipment and improve production efficiency; Promote equipment maintenance to safe and effective condition-based maintenance and state maintenance from the later maintenance and regular maintenance. 


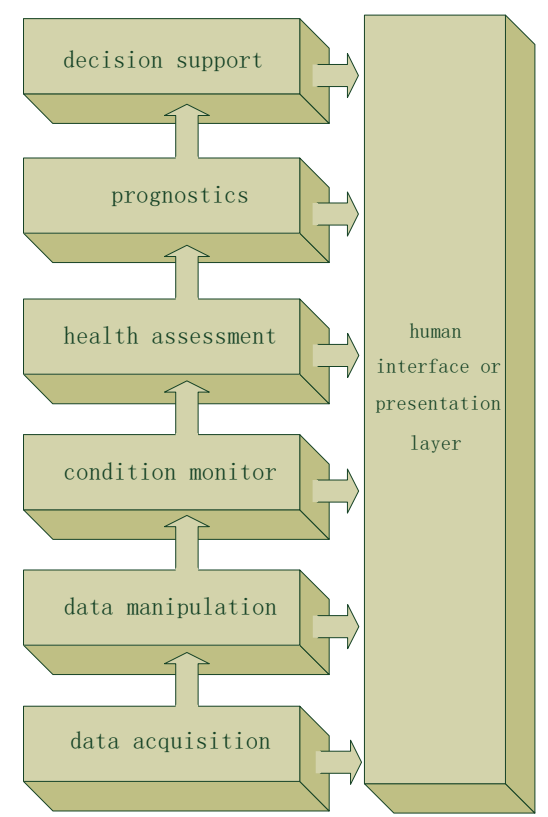

Fig.1. Open system architercture of OSA-CBM

\section{Condition-based Support System of Shore Defense Radar.}

By analyzing the composition, working principle and condition-based support demand of shore defense radar, combined with open system architecture of OSA-CBM, framework of shore defense radar condition-based support system established is shown in figure 2.

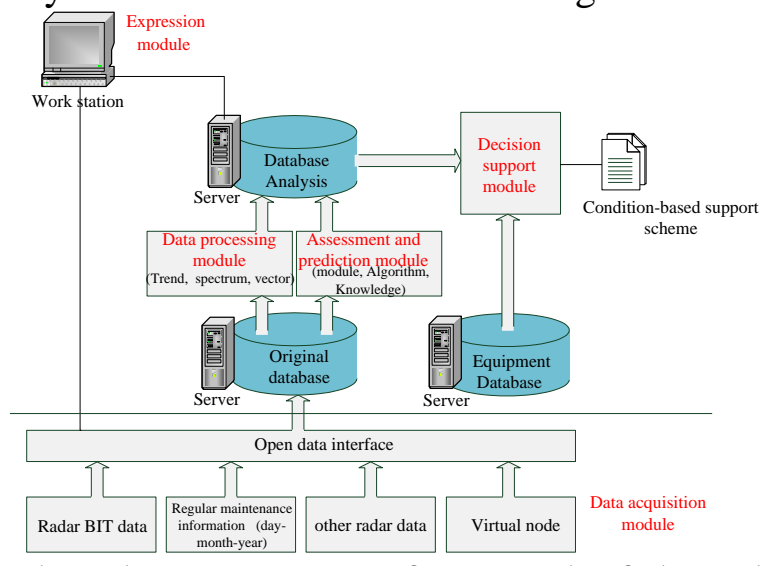

Fig.2. Condition-based support system framework of shore defense radar

Corresponding to the seven layers function system of OSA-CBM, condition-based support system of shore defense radar is divided into the following levels:

1) Data acquisition module

The main data sources of shore defense radar include BIT data under the working state of radar, regular maintenance (day, month, year) information, other radar working condition information and virtual node information.

2) Data processing module

Process the various data achieved by data acquisition modules, make it to process the original data by using filtering or spectrum analysis and transfer it to data or status to identify by the software easily; Meet the needs of establishing system model, diagnosis model and prediction model; Extract the data contained in the failure information, identify potential hidden fault characteristics.

3) Assessment and prediction module

Use the information in the original database and expert knowledge base, set up fault data to make a comprehensive fault diagnosis based on the long-term accumulation of limited data; Apply prediction algorithm, according to the analysis and forecast of the key signal to find and predict the potential hidden faults and its development trend, form diagnosis conclusion and prediction advice 
for use staff query.

4) Decision support module

Through the effective comprehension of various original information, analyzed data, diagnosis and prediction results of shore defense radar, combine the content of spare parts, acquisition flow and maintenance processes in the equipment database, form the decision file guiding the actual equipment maintenance operation. The decision includes optimization decision strategy, optimal maintenance plan and reengineering maintenance process.

5) Expression module

Analysis and query, diagnosis and prediction of data, decision support process of artificial participation and interactive display, all need expression module to design and implementation. The design of the expression module is focused on concise, high degree of automation and fast interactive response.

In the above, the composition framework of shore defense radar condition-based support system has significant difference with the standard system architecture of OSA-CBM, which is mainly embodied that the function of state monitoring is weakened. Working mode of shore defense radar is vehicle, all-weather, adapt to the environment, which determines whether the equipment size, volume and power, all should not increase the complexity of the shore defense radar; BIT data of shore defense radar has realized the abnormal alarm shown in the display control unit, it is not necessary to create online condition monitoring function alone. Therefore, through reasonable design of expression module, query and manage the data and information of condition-based support system of shore defense radar does not increase condition monitoring functions additionally.

\section{Technical difficulties analysis}

\section{The correlation analysis of fault mode and testing parameters.}

Based on detecting signal, implement the objective, reasonable and accurate assessment and prediction for the performance and state of the shore defense radar is the basis of implementing condition-based support. To achieve this purpose, need to deeply analyze the relationship between the fault mode of shore defense radar and the corresponding prediction signal; describe this relationship through procedural description language. Build system fault mode and prediction model based on the data driven mode. Therefore, the correlation analysis technology of fault mode and prediction signal is one of the difficulties to realize condition-based support of shore defense radar.

\section{Diagnosis and prediction method based on limited data.}

Currently, there is a little of diagnosis, prediction theory and algorithm used in the engineering, and is used widely in the aviation, aerospace, Marine, military industry equipment, high-speed and military fields. As an important part of shore defense radar implementation, based on the limited data, constructing the reasonable performance evaluation system, establishing the fault diagnosis and forecast model, choosing suitable fault diagnosis and prediction algorithm are the important technology premise that shore defense radar condition-based support efficiency is give full play.

Fielding troops of shore defense radar has been used for many years, accumulates a wealth of expertise and human experience, which establishes a good foundation for building expert knowledge base of shore defense radar. However, lack of deep and direct shore defense radar detection data is one of the existing short boards that greatly reduces the role of fault diagnosis algorithm and prediction algorithm based on data driven can play, such as neural network reasoning, intelligent diagnosis method based on model and knowledge.

Under this condition, use the preferred fault reasoning method based on the facts (case), combine with the fuzzy evaluation of graded measures, make comprehensive analysis for all kinds of information of the shore defense radar and establish the diagnosis and forecast model based on limited data to realize performance evaluation of the shore defense radar from the qualitative to the quantitative development. Meanwhile, accumulate the BIT data, daily maintenance data and regular 
testing data of shore defense radar, carry out data mining and analysis deeply, and use long-term data to train and optimize the fault model and predictive model to constantly enrich and improve the diagnosis and prediction functions.

\section{Technology outlook}

Condition-based maintenance is more practical than the traditional way. It can carry out maintenance on the equipment timely and moderately and realize controllable maintenance; it reduces the cost of maintenance support, improves the combat readiness and mission success, and has an important significance to improve the battle effectiveness.

Shore defense radar equipment is still in the high speed development in our country; there are many deficiencies. In order to play the role of condition-based maintenance more effectively and achieve the purpose of the "Good application and proper application ", it still needs to focus on developing from the following aspects:

Focus on the testability design of Shore defense radar.

Although the new generation of shore defense radar is designed machine test (BITE) method, and its design is not enough to satisfy the need of conducting in-depth condition assessment and performance prediction. In the process of new type radar design, should continue to improve the testability design level, cover every unit of the main fault modes of shore defense radar and reserve LRU level testing interface, easy to external test equipment to extract relevant information and accurately predict performance assessment and diagnosis.

Establish and perfect fault pattern and expert knowledge base of shore defense radar.

Deeply analyze the fault mode and fault transmission mode of shore defense radar, to form self components level, functional circuit level, SRU level, LRU to system level of fault transmission tree. Meanwhile, improve the expert knowledge base, enrich and perfect a variety of expert knowledge base based model, measured data and cases and lay a foundation for further diagnosis positioning.

\section{Conclusion}

This article started from the principle, interface and data characteristics of shore defense radar, draw lessons from foreign advanced experience in the field of equipment maintenance support, based on the open system architecture of OSA -CBM, designed the overall framework and hierarchy of shore defense radar condition-based maintenance support system. It analyzed the technical difficulties, gave technology outlook on how to improve the condition-based maintenance ability of shore defense radar and carried on the beneficial exploration for the improvement of shore defense radar maintenance support ability in our country.

\section{References}

[1] Yiliu Tu, Xiaodong Li. Industrial Engineering and Management:Future Research and Development in Maintenance Engineering and Management Vol 4(2004) , p. 7-12

[2] Xiaoning Sun. Research on maintenance support pattern and application of the new radar based on process reengineering(National University of Defense Technology ,Changsha 2008)

[3] Yongcheng $\mathrm{Xu}$, Xisen Wen, Xiaoshan Yi, etal. Measurement and control technology: Analysis on the technology development trend of Machine testing Vol20(8) (2001) , p.37-40

[4] Gang Lu, Bing Chen. Radar principle and maintenance of a Certain Type of moving target reconnaissance and spotting(National Defense Industry Press, Beijing 2014)

[5] Ruichao Wang, Yuanda Wang, Junqiang Guo. National Defense Technology: CBM+:A New Trend of Aviation Maintenance Support Vol 1 (2009)1, p.13-14 
[6] Shasha Ma, Xisheng Jia, Lianghua Xia. Journal of the Academy of Equipment Comman\& amp Technology: Study on Condition-based Maintenance for Military Equipment Vol 19(2) (2008), p.112-114 